\title{
Linear free energy relationship in reactions between diphenyl amine and benzyl bromides
}

\author{
S RANGA REDDY and P MANIKYAMBA* \\ Department of Chemistry, Kakatiya University, Warangal 506009 \\ e-mail: mani_prerepa@yahoo.co.in
}

MS received 20 September 2005; revised 24 April 2006

\begin{abstract}
Rate of reaction between benzyl bromide and diphenyl amine is retarded by electron-donating groups and enhanced by electron-withdrawing groups present on the benzene ring of the substrate. Hammett's reaction constant $\rho$ of the reaction decreases with increase in temperature according to the equation,
\end{abstract}

$\rho=-11 \cdot 92+3 \cdot 54 / T$.

Minimal structural effects observed are attributed to the fact that the isokinetic temperature of the reaction is close to the reaction temperature.

Keywords. Diphenyl amine; substituent effect; reaction constant; isokinetic temperature; linear free energy relationship.

\section{Introduction}

Nucleophilic substitution at the benzylic carbon is of broad synthetic utility and has received considerably significant attention from organic chemists. Fundamental concepts like steric effects, nucleophilicity, solvent effects and structure reactivity correlations have been developed. Literature survey shows that a variety of nucleophiles, namely thiourea, ${ }^{1}$ thiobenzamide, ${ }^{2}$ pyridines, ${ }^{3}$ quinolines ${ }^{3}$, different aliphatic and aromatic primary and secondary amines, ${ }^{4} 2$ mercapto benzimidazole ${ }^{5}$ and 2-mercaptobenzoxazole, ${ }^{6}$ have been used in these studies. As part of our broad programme on the benzylation of secondary amines hither to not reported in the literature, we studied the effect of solvents, ${ }^{7}$ on the benzylation of diphenyl amine using benzyl bromide. In continuation of this, the present report deals with the effect of the substituent attached to the benzene ring of benzyl bromide on the rate of benzylation of diphenyl amine.

\section{Experimental}

Benzyl bromide (Riedel), $p$ - $\mathrm{NO}_{2}, p-\mathrm{CH}_{3}, p$-Br benzyl bromides (Fluka) and diphenyl amine (BDH, AR)

*For correspondence were used without further purification. Solvent methanol (Sd-fine) was used after distilling. Temperature of the reaction mixture was maintained constant using a thermostat (INSREF make) with an accuracy of $\pm 0.5^{\circ} \mathrm{C}$. Progress of the reaction was followed by measuring the conductance of the reaction mixture at different time intervals. Solutions of diphenyl amine of appropriate concentrations were prepared by dissolving weighed quantities in methanol medium. Solutions of benzyl bromides in methanol were prepared as required just $30 \mathrm{~min}$ before starting the reaction. The possibility of these benzyl bromides undergoing solvolysis was checked by conducting an independent study. These solvolysis rate constants of $p-\mathrm{CH}_{3}, p-\mathrm{Br}, p-\mathrm{NO}_{2}$ and unsubstituted benzyl bromides in methanol medium were found to be $19 \cdot 19,9 \cdot 21,8.44$ and $12.82 \times 10^{-6} \mathrm{~s}^{-1}$ respectively at $313 \mathrm{~K}$. These values are about 100 times less than the substitution rate constants. Therefore these solvolysis rates are not taken into consideration while studying the substitution reactions. During the course of the reaction the conductance of the reaction mixture increases due to the liberation of $\mathrm{Br}^{-}$which has been identified. Therefore the reactions are followed by measuring the conductance of the reaction mixture at different time intervals. To establish the order with respect to [diphenylamine] the reactions are studied at 
$0.02 \mathrm{~mol} \mathrm{dm}^{-3} \mathrm{~s}^{-1}$ diphenylamine and $0.20 \mathrm{~mol} \mathrm{dm}^{-3} \mathrm{~s}^{-1}$ benzyl bromide. The plot of $\log \left[\left(C_{\infty}-C_{t}\right) / \mathrm{C}_{\infty}\right]$ is linear with negative slope suggesting that the order with respect to the nucleophile is one according to the equation ${ }^{8}$,

$$
k_{\mathrm{I}}=(2 \cdot 303 / t) \log \left[\left(C_{t}-C_{0}\right) /\left(C_{\infty}-C_{t}\right)\right],
$$

where $C_{0}, C_{t}$ and $C_{\infty}$ are the conductances of the reaction mixture at the beginning, after time $t$ and after completion of reaction.

The pseudo first-order rate constants are determined from the slope of this plot. These rate constants, determined at $30^{\circ} \mathrm{C}$ in methanol medium, are $2.47 \times$ $10^{-4} \mathrm{~s}^{-1}, 4.62 \times 10^{-4} \mathrm{~s}^{-1}$ and $9.42 \times 10^{-4} \mathrm{~s}^{-1}$ at $0.1 ; 0 \cdot 2$ and $0.4 \mathrm{~mol} \mathrm{dm}^{-3}$ benzyl bromide respectively. The data confirm the first-order nature of the reaction with respect to [benzyl bromide]. Since the reaction is overall second order, the reactions are studied using [diphenylamine $]=[$ benzyl bromide $]=0.02 \mathrm{~mol} \mathrm{dm}^{-3}$.

Second-order rate constants $k_{\text {II }}$ have been computed from the slopes of the linear plots obtained when $C_{\infty} /\left(C_{\infty}-C_{t}\right)$ is plotted against time according to the equation, ${ }^{8}$

$$
k_{\mathrm{II}}=(1 / \mathrm{at})\left[C_{\infty} /\left(C_{\infty}-C_{t}\right)\right]
$$

where $a$ is the initial concentration of the reactants. A typical second-order plot is shown in figure 1 .

The product that separated at the end of the reaction was identified as the corresponding benzyldiphenyl amine from its IR spectral analysis. This benzyl derivative has a melting point of $142^{\circ} \mathrm{C}$. Its IR spectrum does not show any sharp absorption band around $3200 \mathrm{~cm}^{-1}$ due to the $\mathrm{N}-\mathrm{H}$, while this band is observed in the reactant. Further, the absorptions around

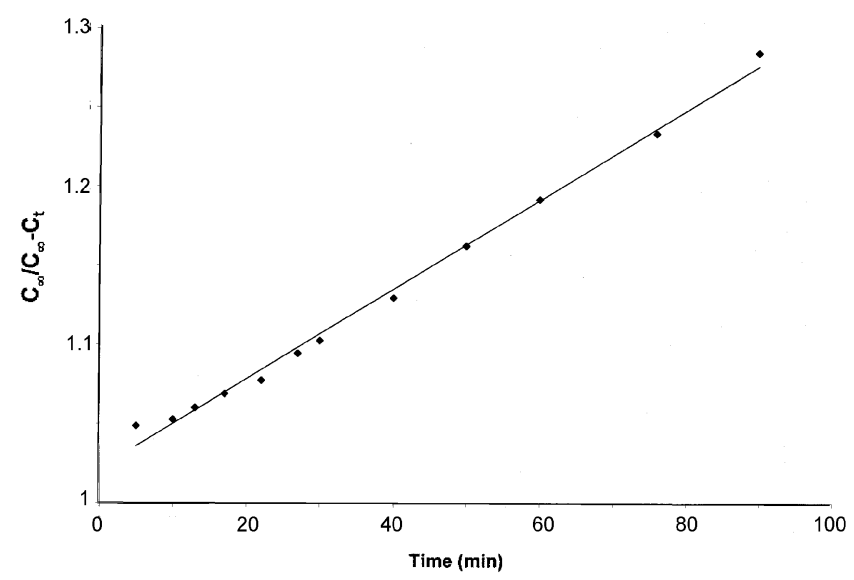

Figure 1. Plot of $C_{\infty} /\left(C_{\infty}-C_{t}\right)$ vs time.
$2800 \mathrm{~cm}^{-1}$ and $1460 \mathrm{~cm}^{-1}$ confirm the presence of the $\mathrm{N}-\mathrm{CH}_{2}$ group. ${ }^{9}$

\section{Results and discussion}

The reaction between benzyl bromides and diphenyl amine has been studied at temperatures $298-313 \mathrm{~K}$ in methanol and the second-order rate constants $\left(k_{\mathrm{II}}\right)$ have been computed. These values, presented in table 1 , indicate that the structural effects operating on the system are minimal. Electron-withdrawing groups $-\mathrm{NO}_{2}$ and $-\mathrm{Br}$ at the para position to $-\mathrm{CH}_{2} \mathrm{Br}$ group enhance the rate of the reaction while the electrondonating $-\mathrm{CH}_{3}$ group retards the rate compared to the unsubstituted compound. To verify the applicability of Hammett's linear free energy relationship (LFER), ${ }^{10}$ $\log k_{\mathrm{II}}$ is correlated with Hammett's substituent constant $\sigma^{11}$ in the temperature range $298-313 \mathrm{~K}$. The following correlations are obtained.

$$
\begin{aligned}
\log k_{\mathrm{II}}= & 1.13+0.64 \sigma, \text { at } 298 \mathrm{~K}, r=1.00(0.004) ; \\
& (0.003) \quad(0.006) \\
\log k_{\mathrm{II}}= & 1.34+0.48 \sigma, \text { at } 303 \mathrm{~K}, r=0.99(0.015) ; \\
& (0.009) \quad(0.021) \\
\log k_{\mathrm{II}}= & 1.65+0.22 \sigma, \text { at } 308 \mathrm{~K}, r=0.99(0.007) ; \\
& (0.004) \quad(0.010) \\
\log k_{\mathrm{II}}= & 1.92+0.10 \sigma, \text { at } 313 \mathrm{~K}, r=0.97(0.017) \\
& (0.010) \quad(0.023)
\end{aligned}
$$

The values in parentheses are the standard errors of the estimates. The reaction constants, which are measures of the sensitivity of the reaction towards the electronic effects of the substituents, obtained from these analyses are small $(\rho<1.0)$ and positive, thus indicating the direct displacement of the leaving group by the nucleophile. Increase in temperature decreases the reaction constant and around $45-50^{\circ} \mathrm{C}$ there is no effect of substituent on rate. According to Exner, ${ }^{12}$ for a given reaction, $\rho$ is influenced by the temperature according to the following relation,

$$
\rho=A[1-\beta / T],
$$

where $A$ is a constant and $\beta$ is the isokinetic temperature. When $\beta=T, \rho=0$, thus isokinetic temperature is the temperature at which the effect of substituent on rate of reaction vanishes and all the substituted compounds in a given series have the same reactivity. 
Table 1. Effect of substituent on rate constant [benzyl bromide - diphenyl amine system]. $[\mathrm{BZBr}]=[\mathrm{DPA}]=0.02 \mathrm{M}$; solvent: methanol

\begin{tabular}{|c|c|c|c|c|c|c|c|c|c|}
\hline \multirow[b]{2}{*}{$\mathrm{BZBr}$} & \multicolumn{5}{|c|}{$k_{2} \times 10^{4} \mathrm{dm}^{3} \mathrm{~mol}^{-1} \mathrm{~s}^{-1} T(\mathrm{~K})$} & \multirow{2}{*}{$\begin{array}{c}E_{a} \\
\left(\mathrm{~kJ} \mathrm{~mol}^{-1}\right)\end{array}$} & \multirow{2}{*}{$\begin{array}{c}* \Delta H^{\neq} \\
\left(\mathrm{kJ} \mathrm{mol}^{-1}\right)\end{array}$} & \multirow{2}{*}{$\begin{array}{c}* \Delta S^{\neq} \\
\left(\mathrm{JK}^{-1} \mathrm{~mol}^{-1}\right)\end{array}$} & \multirow{2}{*}{$\begin{array}{c}* \Delta G^{\neq} \\
\left(\mathrm{kJ} \mathrm{mol}^{-1}\right)\end{array}$} \\
\hline & $\sigma$ & 298 & 303 & 308 & 313 & & & & \\
\hline$-\mathrm{H}$ & 0.00 & $13 \cdot 26$ & $22 \cdot 83$ & $45 \cdot 32$ & $84 \cdot 72$ & $91 \cdot 01$ & 88.49 & -03 & 89.60 \\
\hline$p-\mathrm{CH}_{3}$ & $-0 \cdot 17$ & $10 \cdot 58$ & $17 \cdot 85$ & $41 \cdot 32$ & 77.96 & $79 \cdot 76$ & $77 \cdot 24$ & -42 & $90 \cdot 15$ \\
\hline$p-\mathrm{Br}$ & 0.23 & $18 \cdot 78$ & $27 \cdot 68$ & $49 \cdot 32$ & $91 \cdot 82$ & $83 \cdot 29$ & $80 \cdot 77$ & -27 & 89.09 \\
\hline$p-\mathrm{NO}_{2}$ & 0.78 & $41 \cdot 61$ & $52 \cdot 72$ & $66 \cdot 82$ & $98 \cdot 65$ & $47 \cdot 89$ & $45 \cdot 37$ & -139 & $87 \cdot 46$ \\
\hline
\end{tabular}

$* T=303 \mathrm{~K}$

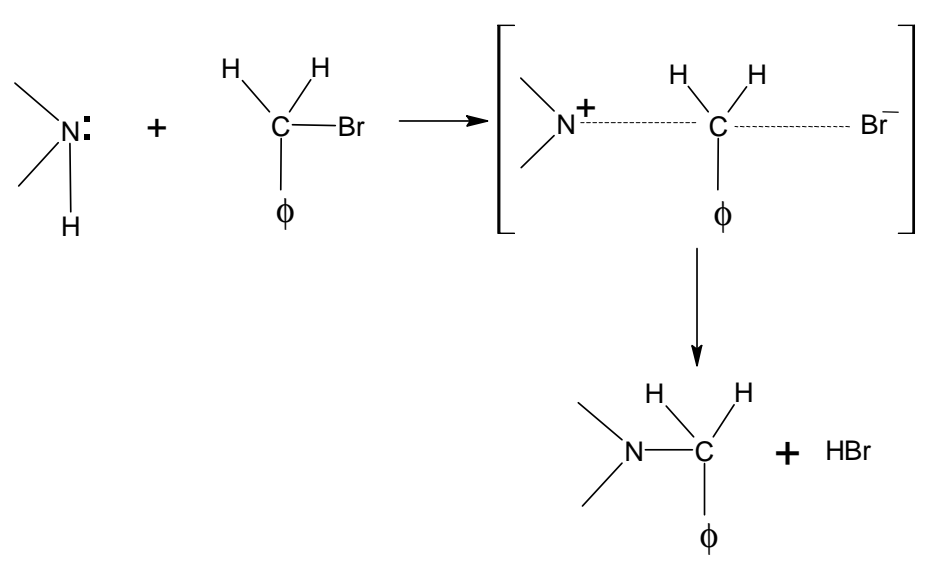

Scheme 1.

activity. In the present case, correlation of $\rho$ with $1 / T$ gives the following equation.

$$
\begin{gathered}
\rho=-11.92+3.54 / T, \quad r=0.99(0.04) . \\
(1.12) \quad(0.34)
\end{gathered}
$$

Thus the isokinetic temperature is $315 \mathrm{~K}$.

From studies of temperature effects on $k_{\mathrm{II}}$ the energy of activation $E_{a}$ and other activation parameters $\Delta H^{\mp}$, $\Delta S^{\ddagger}$ and $\Delta G^{\neq}$are evaluated and recorded in table 1 . These values suggest that there is large variation in $E_{a}$ and $\Delta H^{\neq}$. Though these values are the least for the most reactive $p-\mathrm{NO}_{2}$ benzyl bromide, there appears to be no correlation with Hammetts substitution constant $\sigma . \Delta S^{\ddagger}$ values are negative as expected for reaction systems involving polar transition states. According to Leffler, ${ }^{13}$ for a reaction series which follows Hammett's LFER, the reaction must be either isoentropic or $\Delta S^{ \pm}$should vary linearly with $\Delta H^{ \pm}$according to the equation,

$$
\Delta H^{\ddagger}=\Delta H^{ \pm}{ }_{0}+\beta \Delta S^{ \pm},
$$

where $\beta$ is isokinetic temperature. This correlation between $\Delta H^{\neq}$and $\Delta S^{\neq}$results in the following equation

$$
\begin{gathered}
\Delta H^{ \pm}=89 \cdot 0+316 \cdot 0 \Delta S^{ \pm}, r=0.99(0 \cdot 03) . \\
(1 \cdot 2) \quad(9 \cdot 8)
\end{gathered}
$$

Thus the $\beta$ determined from this correlation is in excellent agreement with value obtained from $\rho-T$ correlation $(315 \mathrm{~K})$. According to Peterson and coworkers ${ }^{14}$ in order for an isokinetic relationship between $\Delta H^{\neq}$and $\Delta S^{\neq}$to be significant, the range of observed $\Delta H^{\neq}\left(\Delta \Delta H^{\ddagger}\right)$ should exceed twice the maximum possible error $(\delta)$ in $\Delta H^{\ddagger}$. In the present system, the calculated values of $\delta \approx 10 \cdot 0 \mathrm{~kJ}$ while $\Delta \Delta H^{\neq}$ is more than $30 \mathrm{~kJ} \mathrm{~mol}^{-1}$ indicating that the $\Delta H^{ \pm}-\Delta S^{\neq}$ correlation is significant. The isokinetic temperature, $\beta$, determined by these two methods is nearer to the temperature at which the reaction is studied. Perhaps this is the reason for a low reaction constant $(\rho<0.7)$ which is a measure of susceptibility of the reaction rate to the electron density changes at the reaction centre. The free energy of activation $\Delta G^{\neq}$is nearly constant $\left(\Delta \Delta G^{\neq}<3.0 \mathrm{~kJ} \mathrm{~mol}^{-1}\right)$ suggesting the operation of similar mechanisms in each series.

Since a positive reaction constant in general indicates a disappearance of positive charge at the reaction center, i.e. benzylic carbon atom in the present 
case, the displacement mechanism shown in scheme 1 may be proposed for the present system.

\section{References}

1. Yoh S D, Lee D S and Hong S Y 1969 J. Korean Chem. Soc. 13215

2. Manikyamba $\mathrm{P}$ and Sundaram E V 1990 Int. J. Chem., Kinet. 221153

3. Padmanabhan V S and Anantakrishnan S V 1975 Curr. Sci. 44187

4. Radhakrishnamurti P S and Panigrahi G P 1970 Bull. Chem. Soc. Jpn. 4381

5. Brown H C and Eldred N R 1949 J. Am. Chem. Soc. 714453
6. Kalyani P, Rajeshwar Rao B and Manikyamba P 2006 J. Chem. Res. (s) accepted

7. Ranga Reddy S and Manikyamba P 2004 Indian J. Chem. A43 1092

8. Frost A A and Pearson R G 1970 Kinetics and mechanism 2nd edn (New Delhi: Wiley Eastern) p. 37

9. Nakanishi K and Solomon P H 1977 Infrared absorption spectroscopy (Holden Day) p. 50

10. Hammett L P 1935 Chem. Rev. 17125

11. Shorter J 1973 Correlation analysis in organic chemistry (Oxford: Clarendon)

12. Exner O 1955 Coll. Czech Chem. Commun. 291094

13. Leffler J E 1955 J. Org. Chem. 201202

14. Peterson R C, Markgraph J H and Ross S D $1961 \mathrm{~J}$. Am. Chem. Soc. 833819 